\title{
Synergistic Inhibitory Effect of Traditional Chinese Medicine Astragaloside IV and Curcumin on Tumor Growth and Angiogenesis in an Orthotopic Nude-Mouse Model of Human Hepatocellular Carcinoma
}

\author{
SHUO ZHANG ${ }^{1 *}$, DECAI TANG $^{1 *}$, WENHUA ZANG $^{2}$, GANG YIN ${ }^{1}$, JIANGUO DAI $^{3}$, \\ YU SUN $^{5}$, ZHIJIAN YANG ${ }^{4,5}$, ROBERT M. HOFFMAN ${ }^{4}$ and XIUXIA GUO ${ }^{1}$ \\ Departments of ${ }^{1}$ Chinese Medicine and ${ }^{3}$ Pathology, School of Basic Medical Sciences, \\ Nanjing University of Chinese Medicine, Nanjing, Jiangsu, P.R. China; \\ ${ }^{2}$ Department of Clinical Chinese Pharmacy, Nanyang Institute of Technology, Nanyang, Henan, P.R. China; \\ ${ }^{4}$ AntiCancer, Inc., San Diego, CA, U.S.A.; \\ ${ }^{5}$ Origin Biosciences Inc., Nanjing, Jiangsu, P.R. China
}

\begin{abstract}
Aim: The aim of the present study was to investigate the efficacy of the traditional Chinese medicine (TCM), astragaloside IV (AS-IV) and curcumin on tumor growth and angiogenesis in an orthotopic nude-mouse model of human hepatocellular carcinoma (HCC). We have previously shown the usefulness of orthotopic models of human cancer for evaluation of the efficacy of TCM. Materials and Methods: Nude mice with orthotopic HepG2 HCC were treated with vehicle control (0.01 $\mathrm{ml} / \mathrm{g}$ normal saline), cisplatinum (2 $\mathrm{mg} / \mathrm{kg}), A S-I V(20 \mathrm{mg} / \mathrm{kg})$, curcumin (100 mg/kg) or AS-IV plus curcumin $(20 \mathrm{mg} / \mathrm{kg}+100$ $\mathrm{mg} / \mathrm{kg}$ ). Tumor inhibition in each group was evaluated by tumor weight at autopsy. The effect of AS-IV and curcumin on tumor angiogenesis was assessed by CD34 staining and expression of fibroblast growth factor-2 (FGF2), matrix metalloproteinase 2 (MMP2), vascular endothelial growth factor (VEGF), hepatocyte growth factor (HGF), thrombosis-related factor tissue factor (TF) and coagulation factor VII (FVII), as well as microRNAs miR-122 and miR-221. Results: AS-IV and curcumin alone and
\end{abstract}

This article is freely accessible online.

*These Authors contributed equally to this work.

Correspondence to: Dr. Decai Tang, School of Basic Medical Sciences, Department of Chinese Medicine, Nanjing University of Chinese Medicine, 138 Xianlin Road, Nanjing, Jiangsu 210023, P.R. China. E-mail: talknow@163.com or Robert M. Hoffman, Ph.D., AntiCancer Inc., 7917 Ostrow Street, San Diego, CA 92111, USA.E-mail: all@anticancer.com

Key Words: Astragaloside IV, curcumin, TCM, hepatocellular carcinoma, nude mouse, orthotopic, angiogenesis, microRNA. in combination significantly reduced mean tumor weight compared to vehicle control $(p<0.05)$. Tumor microvessel count was reduced by $A S-I V$ and curcumin alone. Expression of FGF2, $M M P 2, V E G F, H G F, T F$ and FVII was reduced by $A S-I V$ and curcumin alone. AS-IV and curcumin alone up-regulated expression of miR-122 and down-regulated that of $m i R-221$. The combination of AS-IV and curcumin demonstrated significant synergistic effects on microvessel count as well as on expression of angiogenic and thrombosis-related factors and microRNAs. Conclusion: The present study indicates future clinical potential of combination therapy with AS-IV and curcumin for HCC.

Hepatocellular carcinoma (HCC) is a highly aggressive and hypervascular tumor, associated with high morbidity (1), and is recalcitrant to therapy (2). Targeting angiogenesis could be an effective therapeutic strategy for controlling the progression of HCC.

Traditional Chinese Medicine (TCM) has been widely used for anticancer treatment in China with a long history. Astragaloside IV (AS-IV) is a representative constituent of Astragalus membranaceus (Fisch.) Bge. Var. mongholicus (Bge.) Hsiao and has various pharmacological activities, including anti-tumor, anti-inflammatory and anti-diabetic (3, 4). Curcumin (diferuloylmethane) is the chief component of the spice turmeric and is derived from the rhizome of the East Indian plant Curcuma longa. Curcumin exhibits anti inflammatory, antioxidant and anticancer efficacy (5). AS-IV was reported to reduce invasiveness and angiogenesis of gastric cancer cells and down-regulate the expression of fibroblast growth factor-2 (FGF-2) and suppress angiogenesis of HCT116 colon cancer cells $(6,7)$. Curcumin showed anti-metastatic and anti-angiogenesis efficacy against small-cell lung cancer 
through inhibition of matrix metalloproteinase-2 and -9 (MMP2/-9) activation and down-regulating signal transduction and activator of transcription 3 (STAT3) expression $(8,9)$. However, anti-angiogenic efficacy of AS-IV and curcumin on HCC has not been investigated to our knowledge.

Our laboratories have demonstrated the usefulness of orthotopic nude-mouse models of human cancer for the evaluation of efficacy of TCM herbal mixtures and compounds (10-14).

In the present study, we used a nude-mouse orthotopic liver-cancer model to evaluate the anti-angiogenic and antitumor efficacy of AS-IV and curcumin, alone and in combination. Expression of angiogenesis-related factors and genes in the treated tumors was examined to explore the underlying anti-angiogenic mechanism of these herbal agents.

\section{Materials and Methods}

Cell culture. The human hepatocarcinoma cell line HepG2 was obtained from Yangzhou College, Yangzhou, China and cultured in RPMI-1640 medium (GIBCO, Grand Island, NY, USA) with $10 \%$ calf serum (Tianhang Biotechnology Company, Zhejiang, China) plus $1 \%$ penicillin and streptomycin at $37^{\circ} \mathrm{C}$ in a $5 \% \mathrm{CO}_{2}$ atmosphere. Logarithmic-growth-phase HepG2 cells $\left(5 \times 10^{6}\right)$ were treated with $0.25 \%$ trypsin (GIBCO) and suspended in $200 \mu \mathrm{l}$ serum-free RPMI-1640 for subsequent injection into nude mice

HCC orthotopic mouse model. Male BALB/c nude mice (5-6 weeks old) were purchased from Slack Laboratory Animal Company (Shanghai, China). Animal care was in accordance with institutional animal care guidelines. All mice were maintained in a HEPAfiltered environment at $24-25^{\circ} \mathrm{C}$, and humidity was maintained at $50-60 \%$. All animals were fed with autoclaved laboratory rodent diet. Animal experiments were approved by the Animal Committee of Nanjing Origin Biosciences, China (OR1507).

Stocks of HepG2 tumors were established by subcutaneously injecting HepG2 cells $\left(5 \times 10^{6}\right)$ into the flank of nude mice. The subcutaneous tumors were harvested at the exponential growth phase and resected under aseptic conditions. Necrotic tissues were removed, and viable tissues were cut with scissors and minced into $1 \mathrm{~mm}^{3}$ pieces. For orthotopic tumor implantation, animals were anesthetized by injection of $0.02 \mathrm{ml}$ of solution of $50 \%$ ketamine, $38 \%$ xylazine, and $12 \%$ acepromazine maleate. The surgical area was sterilized with povidone iodine. A transverse incision was made below the right costal margin. A tumor fragment was transplanted into an incision within the liver parenchyma. To prevent bleeding, the implanted tumor was dabbed with sterile cotton. The abdominal wound was closed with 5-0 silk sutures. All surgical procedures and animal manipulations were conducted under HEPA-filtered laminarflow hoods with a $\times 8$ surgical microscope (Shanghai Precision Instruments, Shanghai, China).

Treatment. Astragaloside IV (AS-IV) (98.3\% purity by highperformance liquid chromatography [HPLC]) and curcumin $(98.2 \%$ purity by HPLC) were obtained from Zelang Medical Technologicy (Nanjing, China). Cisplatinum was purchased from Qilu Pharmaceutical (Shandong, China). The mice were randomly divided into five groups of eight after the third day of orthotopic tumor implantation. The vehicle-control group was given saline via oral gavage at $0.01 \mathrm{ml} / \mathrm{g}$. The cisplatinum group was treated by intraperitoneal injection at a dose of $2 \mathrm{mg} / \mathrm{kg}$. The AS-IV group was treated via oral gavage at a dose of $20 \mathrm{mg} / \mathrm{kg}$. The curcumin group was treated via oral gavage at a dose of $100 \mathrm{mg} / \mathrm{kg}$. The combination group (AS-IV plus curcumin) received AS-IV and curcumin via oral gavage at doses of $20 \mathrm{mg} / \mathrm{kg}$ and $100 \mathrm{mg} / \mathrm{kg}$, respectively. The cisplatinum group was treated once every 3 days for 21 days. The control, AS-IV, curcumin and AS-IV plus curcumin groups were treated daily for 21 days. Mouse body weight and clinical signs were recorded over the course of the experiments. All mice were sacrificed 2 days after the last treatment. At autopsy the tumor was removed and weighed. The inhibition of tumor in each group was calculated with the formula: inhibition $(\%)=100 \times($ mean tumor weight of the control group - mean tumor weight of the treated group)/mean tumor weight of the control.

Immunohistochemistry. Tumor tissues collected at autopsy were fixed in $10 \%$ buffered formalin, paraffin-embedded and sliced into $4 \mu \mathrm{m}$ sections for immunohistochemical staining of hematopoieticprogenitor-cell antigen CD34 and vascular-endothelial growth factor (VEGF). Slides were deparaffinized in xylene, followed by rehydration in ethanol. Endogenous peroxidase was blocked by incubation with $3 \%$ hydrogen peroxide. The slides were incubated with primary antibody to CD34 (ab81289, 1:100; Abcam, Cambridge, UK) and to VEGF (ab46154, 1:200; Abcam) at $37^{\circ} \mathrm{C}$ for $2 \mathrm{~h}$. After washing with phosphate-buffered saline, the slides were incubated with the secondary antibody labeled by horseradish peroxidase (HRP) at room temperature for $30 \mathrm{~min}$. The sections were counterstained with Mayer's hematoxylin. The whole slide was first viewed at $\times 100$ magnification in order to identify a 'hot spot' representing the area of the highest vessel density. The field was then switched to $\times 400$ magnification for analysis. The microvessel count (MVC) represented by CD34 staining was calculated as the average number of $\mathrm{CD}_{3} 4^{+}$vessels in four fields of view. The positivelystained areas and the integral optical density (IOD) of VEGF in each field were analyzed by Image-Pro Plus 6.0 software (Media Cybernetics, Silver Spring, MD, USA). The final VEGF value was calculated from five randomly-selected fields of each section using the following formula: mean $\mathrm{OD}$ value $=(\mathrm{IOD} /$ positive area $)$.

Enzyme-linked immunosorbent assay (ELISA). Blood was collected and serum was prepared for all mice at the end of the study. The serum FGF-2 and MMP-2 levels were determined with ELISA kits (Xinbosheng biotechnology, Nanjing, China) according to their instructions. Briefly, the flat-bottom 96-well microtiter plates were coated with anti- FGF-2 and anti-MMP-2 and incubated overnight at $4^{\circ} \mathrm{C}$ then blocked with bovine serum albumin buffer at $37^{\circ} \mathrm{C}$ for $3 \mathrm{~h}$. The wells were then loaded with diluted serum samples or standard $(100 \mu \mathrm{l} /$ well $)$ and incubated at $37^{\circ} \mathrm{C}$ for $2 \mathrm{~h}$. The plates were washed five times and incubated with biotinylated anti-FGF2 and anti-MMP- 2 at $37^{\circ} \mathrm{C}$ for $1 \mathrm{~h}$. After washing five times, the plates were incubated with horseradish-peroxidase-labeled avidin at $37^{\circ} \mathrm{C}$ for $30 \mathrm{~min}$. After washing five times, the plates were incubated with tetramethylbenzidine substrate $(100 \mu \mathrm{l} /$ well $)$ at $37^{\circ} \mathrm{C}$ for $30 \mathrm{~min}$. The color reaction was stopped by the addition of $\mathrm{H}_{2} \mathrm{SO}_{4}$ (2 mol/l, $50 \mu \mathrm{l} /$ well). The optical density (OD) was then measured at $450 \mathrm{~nm}$ using a microplate reader (ELx800uv; Bio-Tec Instruments, Houston, TX, USA). The concentrations of FGF-2 and MMP-2 were calculated according to calibration curve. 
Table I. Primer sequences for real-time polymerase chain reaction (PCR).

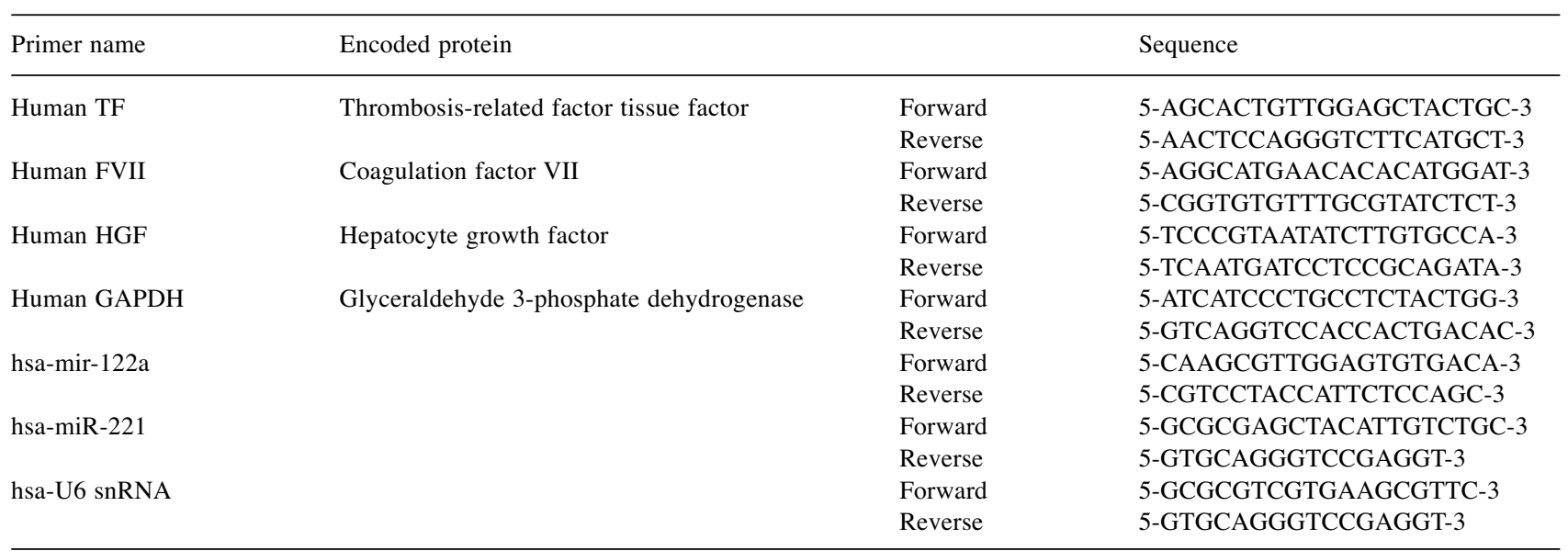

Real-time polymerase chain reaction (RT-PCR). Total RNA was isolated from tumor tissues collected at autopsy in $1 \mathrm{ml}$ TRIzol reagent (Invitrogen Life Tech, Carlsbad, CA, USA). Real-Time PCR was performed in a final volume of $20 \mu \mathrm{l}$ containing $1 \mu \mathrm{l}$ cDNA template, $2 \mu \mathrm{l}$ primer, $10 \mu \mathrm{l}$ of SYBR Green Master Mix (TOYOBO, Japan), and $7 \mu$ diethy pyrocarbonate water (KeyGen BioTech, Nanjing, China). Conditions used for PCR consisted of 40 cycles at $95^{\circ} \mathrm{C}$ for $5 \mathrm{~min}, 95^{\circ} \mathrm{C}$ for $15 \mathrm{~s}, 60^{\circ} \mathrm{C}$ for $20 \mathrm{~s}$, and $72^{\circ} \mathrm{C}$ for $40 \mathrm{~s}$. The melting curve was analyzed at the temperature range of $65-95^{\circ} \mathrm{C}$. Primers were designed as shown in Table I. Glyceraldehyde 3phosphate dehydrogenase $(G A P D H)$ served as an internal reference for thrombosis-related factor tissue factor $(T F)$, coagulation factor VII $(F V I I)$ and hepatocyte growth factor $(H G F)$. U6 snRNA served as an internal references for hsa-mir-122a and hsa-miR-221. Gene expression was calculated using the $2^{-\Delta \Delta \mathrm{Ct}}$ method.

Western blotting. Protein from tumor tissues collected at autopsy was extracted with RIPA buffer (Beyotime BioTech, Shanghai, China). Protein concentration was determined with the Bradford protein assay kit (KeyGen BioTech). Total proteins were separated by sodium saltpolyacrylamide gel electrophoresis and electrophoretically transferred to a polyvinylidene fluoride membrane (Millipore, Bedford, MA, USA). The membranes were incubated with $5 \%$ nonfat milk solution for blocking non-specific binding and with primary antibodies to TF ((ab104513; 1:400), anti-FVII (ab97614; 1:600), anti-HGF (ab178395; 1:800) (Abcam). After washing twice with tris-buffered saline, the membranes were incubated with appropriate secondary antibodies conjugated with HRP for $2 \mathrm{~h}$ at room temperature. Analysis of electro-chemi-luminescence was performed according to the manufacturer's instructions using a Bio-Rad imaging system and Quantity One version software (Bio-Rad, Hercules, CA, USA) was used to quantify the density of bands.

Statistical analysis. All data are presented as the mean values \pm standard deviation and were analyzed using SPSS 17.0 software (Chicago, IL, USA). Comparisons between different groups were conducted using a one-way ANOVA, with least significant difference used for inter-group comparison. A value of $p<0.05$ was considered to indicate a statistically-significant difference.

\section{Results}

$A S-I V$ and curcumin inhibited tumor growth. The effect of AS-IV and curcumin on tumor growth was evaluated in the HepG2 HCC orthotopic mouse model. Tumor weight was significantly reduced by cisplatinum, AS-IV, curcumin, and AS-IV plus curcumin as compared to the vehicle control $(p<0.05)$ (Figure 1). A greater inhibitory efficacy on tumor growth was observed with the AS-IV plus curcumin combination than AS-IV or curcumin alone, although statistical significance was not achieved $(p>0.05)$ (Figure 1).

$A S-I V$ and curcumin reduced tumor vascularization. As shown in Figure 2A, CD34-positive staining was indicated by the presence of brown particles. In the vehicle-control group, there was a notable increase in CD34-positive staining in tumor tissues and the capillary networks were misaligned, tortuous and irregular in the tumor tissues of all groups. Decreased MVC was found in AS-IV, curcumin and AS-IV plus curcumin combination groups compared with the vehicle control and cisplatinum groups $(p<0.01)$. The combination-treated group showed a significantly greater decrease in MCV than did the groups treated with AS-IV, or curcumin alone $(p<0.05)$, indicating synergistic antiangiogenic efficacy of the AS-IV and curcumin combination (Figure 2B).

$A S-I V$ and curcumin down-regulated expression of angiogenic factors. The expression of VEGF in tumors of all treatment groups was significantly down-regulated compared with those of the vehicle control $(p<0.01)$. The lowest VEGF expression was found in the AS-IV-curcumin combination group compared to the cisplatinum-, AS-IV-, and curcumintreated groups of mice $(p<0.01)$ (Figure $3 \mathrm{~A}$ and $\mathrm{B})$. 

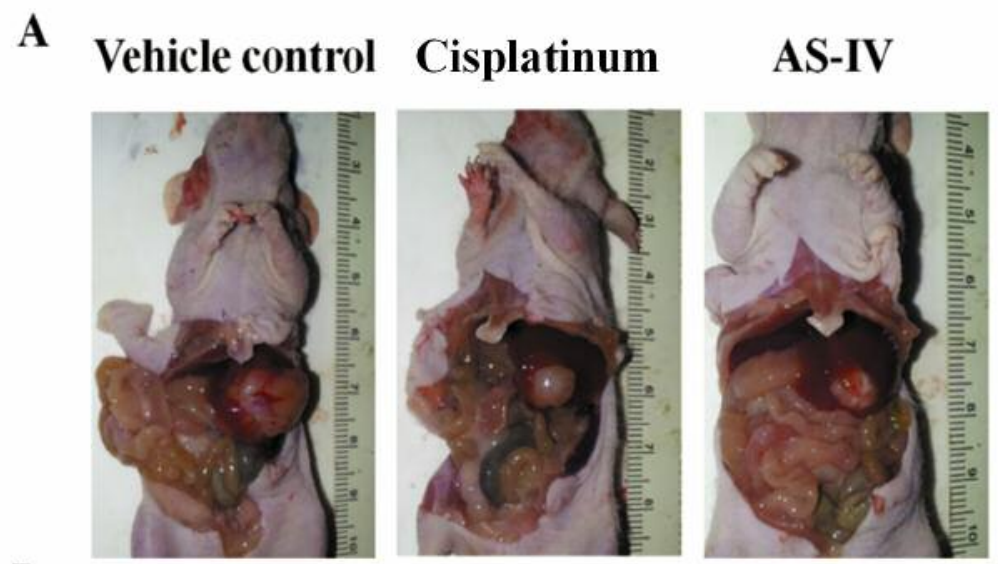

\section{Curcumin}

AS-IV+

B
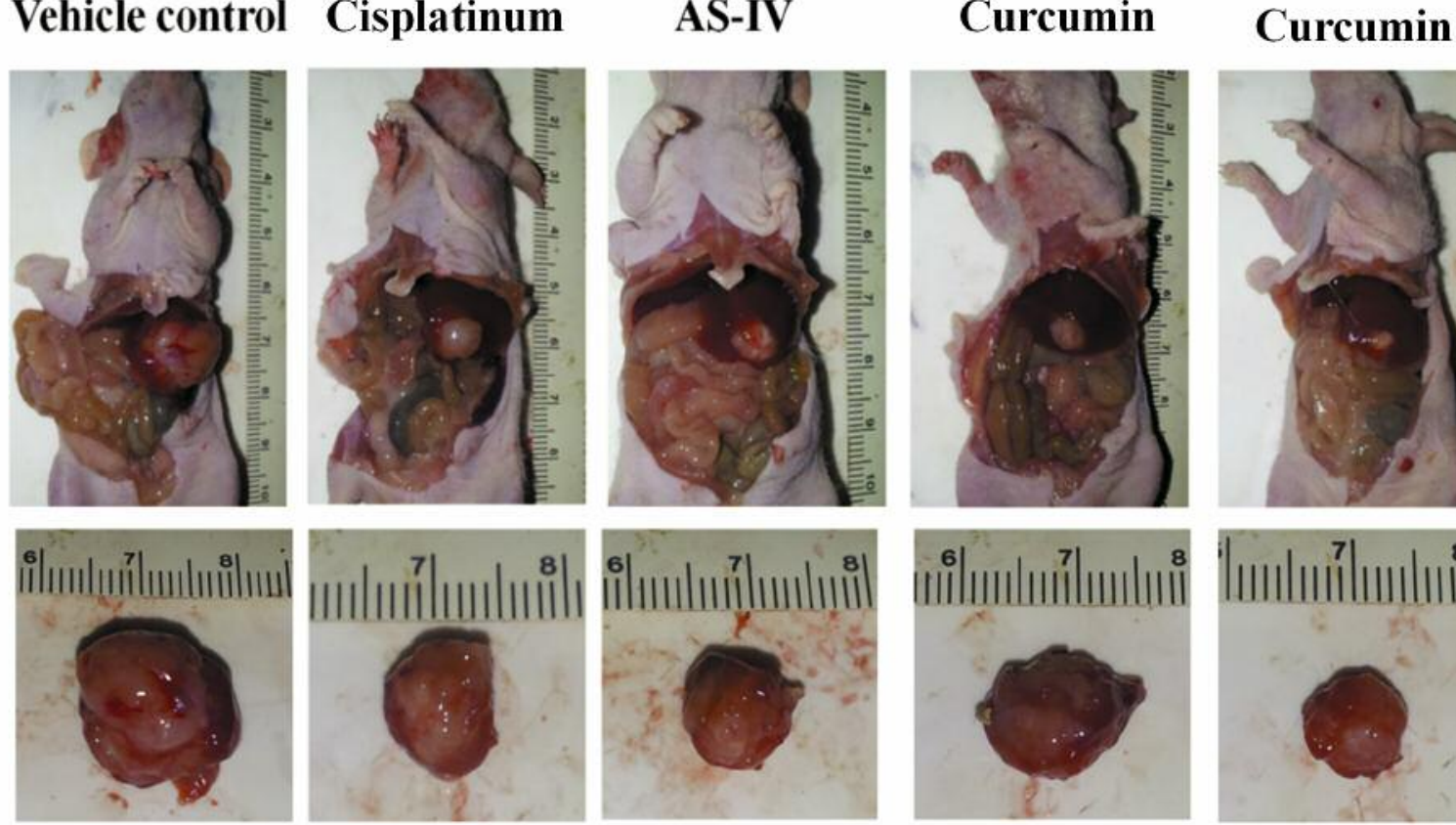

C

D
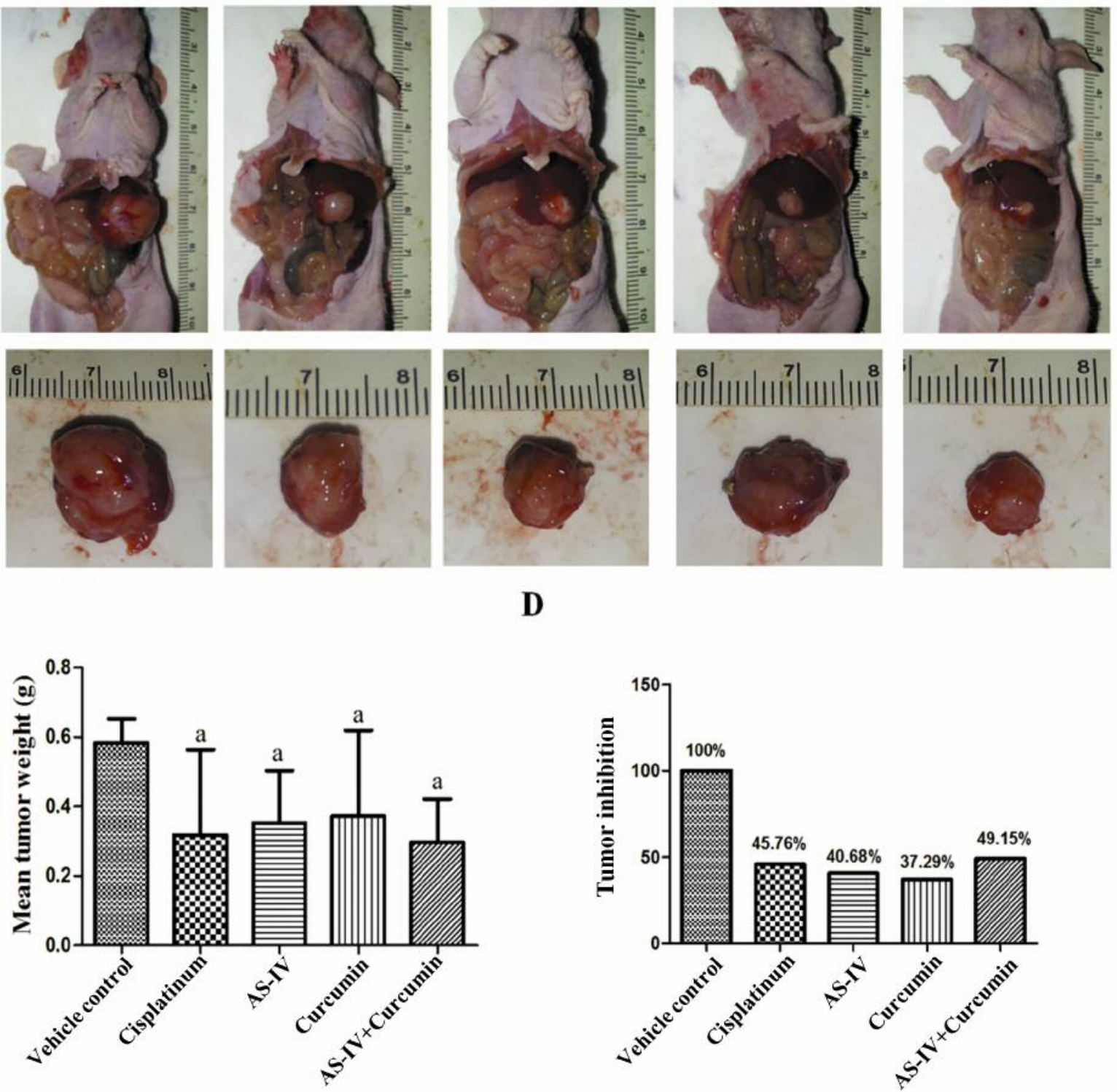

Figure 1. Efficacy of astragaloside IV (AS-IV) and curcumin on tumor growth in the HepG2 HCC orthotopic nude-mouse model. A: Representative orthotopic liver tumors of each treated group at autopsy. B: Representative excised liver tumor of each treated group at autopsy. C: Mean tumor weight of each treated group. Results are presented as the mean $\pm S D(n=8)$. a Significantly different at $p<0.05$ vs. vehicle control. D: Percentage of tumor growth of each treated group relative to control.

The serum levels of FGF-2 and MMP-2 were significantly reduced in all treatment groups compared with the vehicle control $(p<0.01)$.The serum FGF-2 and MMP2 levels in groups treated with AS-IV, curcumin, and AS-IV plus curcumin groups were lower than in the cisplatinum-treated group $(p<0.01)$. The AS-IV-curcumin combination had the most effective inhibition of serum
FGF-2 and MMP-2 among all treated groups $(p<0.01)$ (Figure 3C and 3D).

HGF mRNA and protein expression was down-regulated by all treatments as compared with the vehicle control $(p<0.01)$. The AS-IV plus curcumin combination-treated mice had significantly lower HGF mRNA expression than the other treated groups $(p<0.01)$ (Figure 3E-G). 
$A S-I V$ and curcumin inhibited the expression of thrombosisrelated factors. TF and FVII mRNA expression were downregulated by cisplatinum, AS-IV, curcumin, and the combination of AS-IV with curcumin compared with the vehicle control $(p<0.01)$ (Figure 4A).

The combination-treated group had significantly lower TF and FVII mRNA expression levels than the cisplatinum, ASIV, and curcumin groups $(p<0.01)$ (Figure $4 \mathrm{~A})$. AS-IV, curcumin, and the combination AS-IV+ curcumin decreased $\mathrm{TF}$ and FVII protein expression compared with the vehicle control $(p<0.01$. Significantly decreased TF and FVII protein expression was found in the AS-IV plus curcumin combination group compared with the cisplatinum- and ASIV-treated groups $(p<0.05)$ (Figure 4B and C).

The combination of AS-IV and curcumin up-regulated miR122 expression and down-regulated miR-221 expression. The cisplatinum-, AS-IV-, and curcumin-treated groups did not show significant differences in $m i R-122$ expression as compared with the vehicle control group $(p>0.05)$. However, treatment with the AS-IV-curcumin combination significantly up-regulated the expression of miR-122 $(p<0.01)$ (Figure 5A). $m i R-221$ expression was significantly down-regulated in all treatment groups compared with the vehicle control $(p<0.01)$. The AS-IV-curcumin combination group showed a greater decrease in $m i R-221$ expression than did the other treatment groups $(p<0.01)$ (Figure 5B).

\section{Discussion}

The aim of the present study was to investigate the efficacy of AS-IV and curcumin on the inhibition of tumor growth and angiogenesis in an orthotopic nude-mouse model of HCC. A new finding of our study is that AS-IV and curcumin in combination had a synergistic effect for treatment of HCC, leading to enhanced inhibition of tumor growth and angiogenesis in a mouse model of HCC. When examined for the mechanism, we found that this synergistic efficacy of AS-IV and curcumin was associated with regulation of some angiogenesis-related factors and microRNAs.

Angiogenesis is a dynamic process involving several key factors possessing pro-angiogenic activity. VEGF stimulates endothelial-cell proliferation and induces budding in neovascularization (15). FGF-2 binds to heparin sulfate proteoglycans initially, and then the complex binds to the FGF receptor, leading to proliferation and angiogenesis $(16,17)$. MMP-2 not only degrades type IV collagen, but also induces cellular proliferation and angiogenesis (18). HGF is a stromal-cell factor able to promote tumor angiogenesis through stimulation of vascular endothelial cell proliferation, migration and activation of the protein kinase B and extracellular signal-

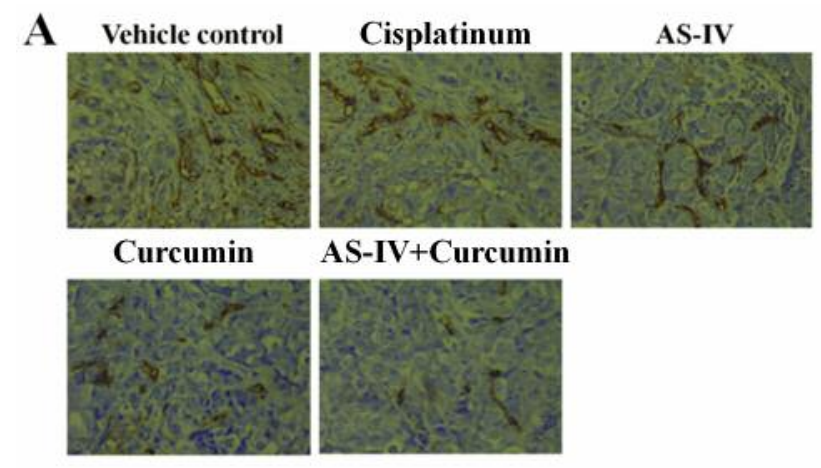

B

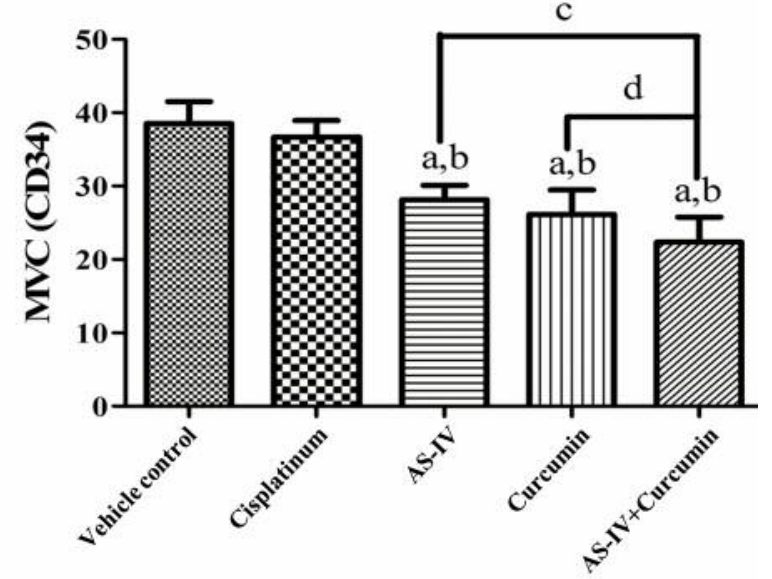

Figure 2. Efficacy of astragaloside IV (AS-IV) and curcumin on tumor vascularization in the HepG2 HCC orthotopic nude-mouse model. A: Representative immunohistochemical staining for CD34 in the tumor at $\times 400$ magnification. B: Microvessel count (MVC) of each treated group. Results are presented as the mean $\pm S D$. Significantly different at $p<0.01$ vs. ${ }^{a}$ vehicle control, ${ }^{b}$ cisplatinum, ${ }^{c} A S-I V$ and $d_{\text {curcumin }}$ groups.

regulated kinases. Auyeung et al. (19) found that Astragalus saponins modulated angiogenesis in human gastric adenocarcinoma cells through inhibition of VEGF and MMP-2 protein. Di et al. (20) demonstrated that curcumin inhibited the transcript levels of two major angiogenesis factors, VEGF and FGF-2, in human breast cancer cells. Our results were consistent with these reports but demonstrated a greater inhibitory effect of AS-IV and curcumin in combination on these angiogenic factors than AS-IV or curcumin treatment alone.

TF and FVII have been reported to contribute to tumor angiogenesis and metastasis progression by initiating the exogenous coagulation pathway in some cancer types (2123). In this study, we show for the first time that suppression of TF and FVII may be involved in the anti-angiogenesis efficacy of AS-IV and curcumin.

MicroRNAs belong to an abundant family of endogenous, short and non-coding RNAs. MicroRNAs that have 
$\mathbf{A}$

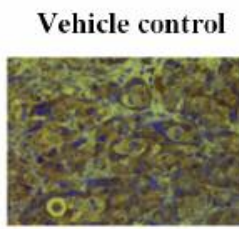

Curcumin

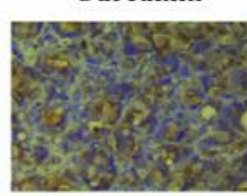

AS-IV+Curcumin

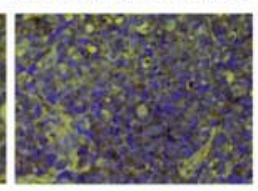

C

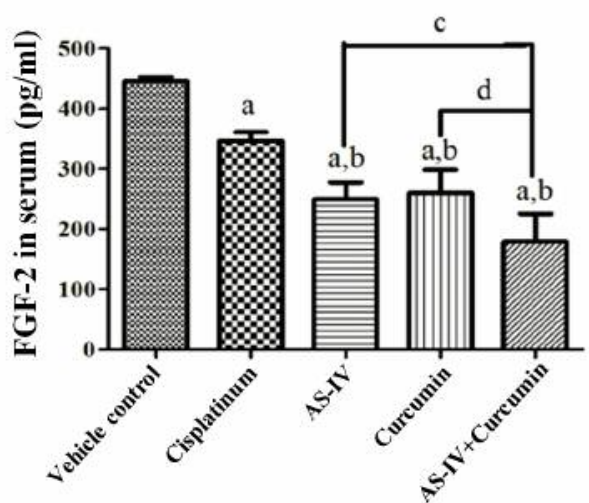

E

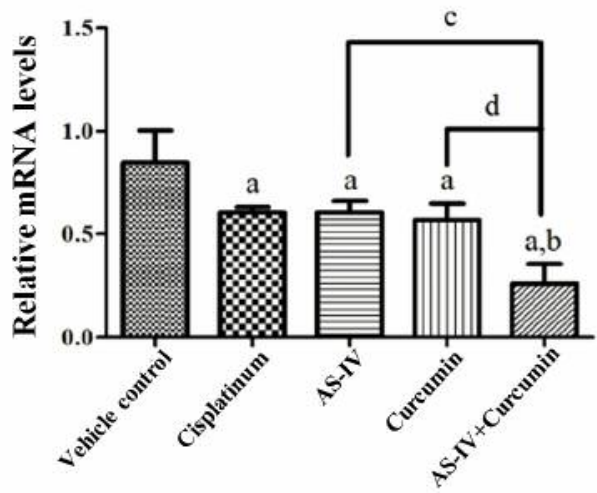

B

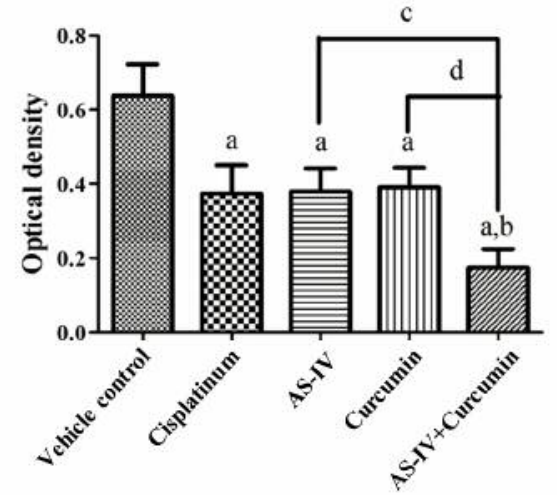

D

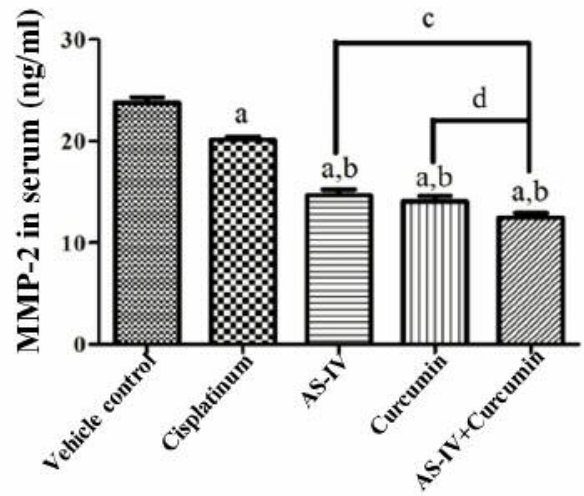

F

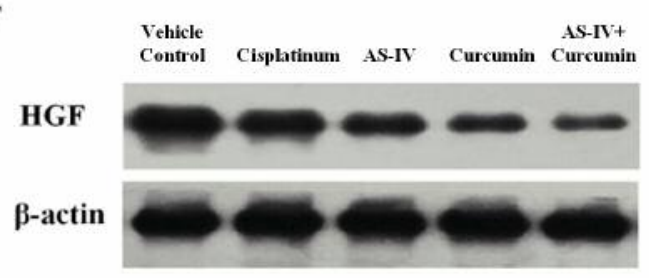

G

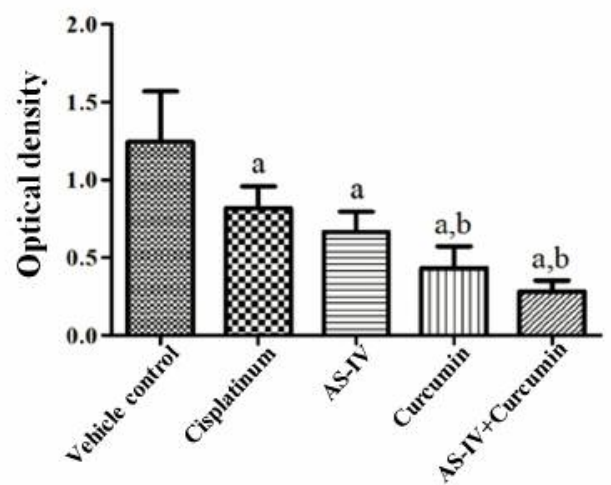

Figure 3. Efficacy of astragaloside IV (AS-IV) and curcumin on the expression of angiogenic factors. A: Representative immunohistochemical staining for vascular endothelial growth factor (VEGF) in the tumor at $\times 400$ magnification. B: Optical density of immunohistochemistry staining for VEGF. $C, D$ : Serum fibroblast growth factor-2 (FGF-2) (C) and matrix metalloproteinase-2 (MMP-2) (D) level in each treated group. E-G: hepatocyte growth factor $(H G F)$ mRNA $(E)$ and protein $(F, G)$ expression in each treated group. Results are presented as mean $\pm S D$. Significantly different at $p<0.01$ vs. ${ }^{a}$ vehicle control, ${ }^{b}$ cisplatinum, ${ }^{c} A S-I V$ and ${ }^{d}$ curcumin groups. 
$\mathbf{A}$

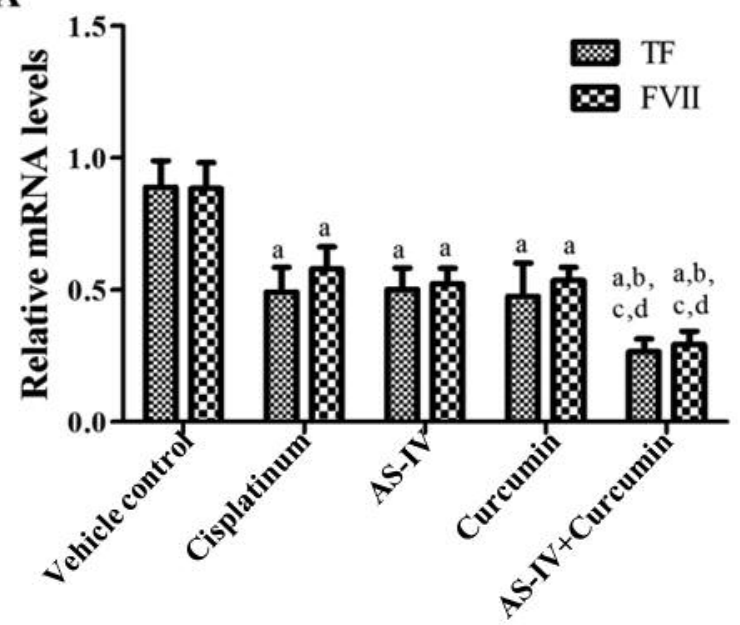

B

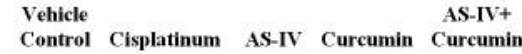

TF

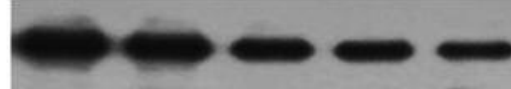

FVII

$\beta$-actin

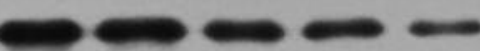

C

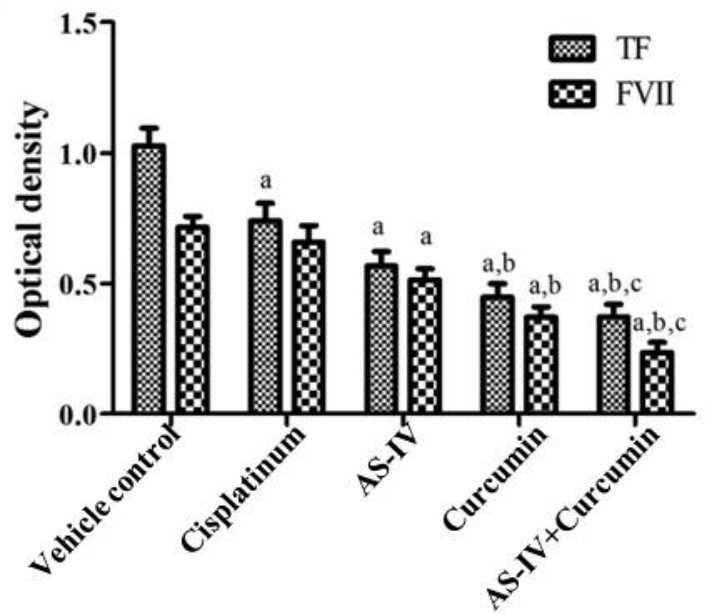

Figure 4. Efficacy of astragaloside IV (AS-IV) and curcumin against the expression of thrombosis-related tissue factor (TF) and coagulation factor VII (FVII). mRNA $(A)$ and protein expression $(B, C)$ of TF and FVII in each treated group. Results are presented as the mean \pm SD. Significantly different at $p<0.01 \mathrm{vs}$. ${ }^{a}$ vehicle control, ${ }^{b}$ cisplatinum and ${ }^{c} A S-I V$ groups.

$\mathbf{A}$

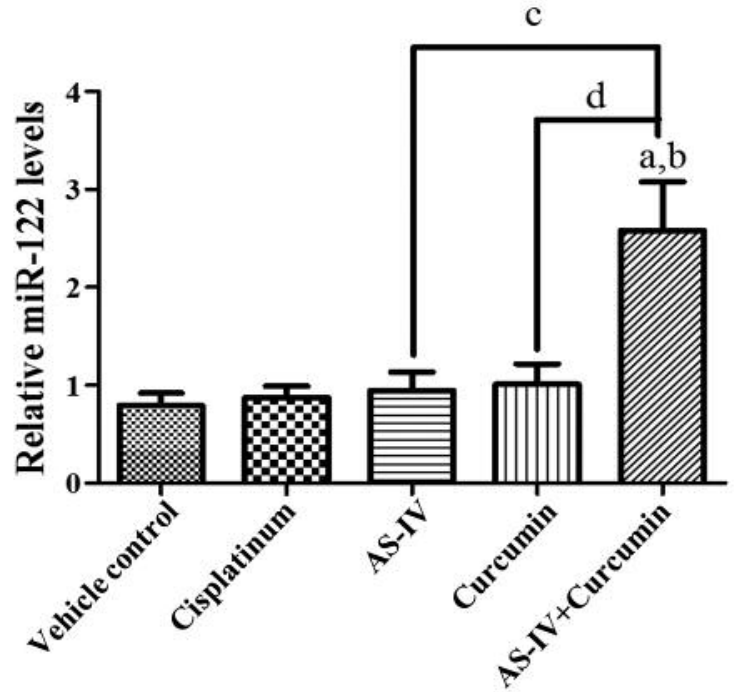

B

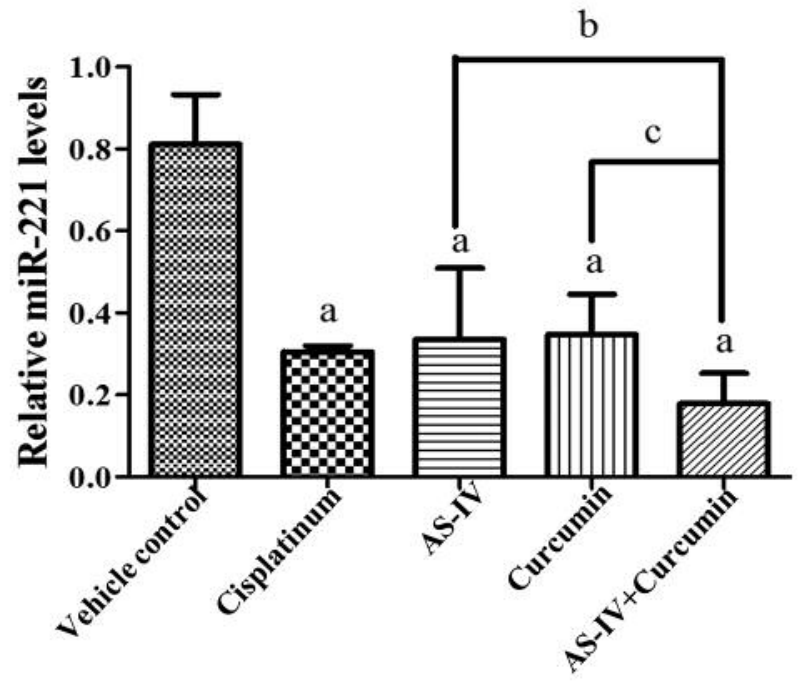

Figure 5. Efficacy of astragaloside IV (AS-IV) and curcumin on the expression of miR-122 and miR-221. A: Tumor miR-122 expression in each treated group. Results are presented as the mean $\pm S D(n=5)$. Significantly different at $p<0.01$ vs. ${ }^{a}$ vehicle control, ${ }^{b}$ cisplatinum, ${ }^{c} A S-I V$ and ${ }^{d}$ curcumin groups. B: Tumor miR-221 expression in each treated group. Results are presented as the mean $\pm S D(n=5)$. Significantly different at $p<0.01 v s$.

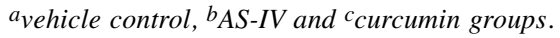


oncogenic or tumor-suppressive properties play an important role in cancer onset and progression. $m i R-122$, as a tumorsuppressor gene, accounts for approximately $70 \%$ of the total miRNA in the liver (24) and inhibits metastasis of HCC through anti-angiogenic activity (25). $m i R-221$ acts as an oncogene in HCC and is essential for angiogenesis by $\mathrm{NOTCH}$ signaling and induces proliferation and migration of endothelial cells (26). Our result suggests that up-regulation of $m i R-122$ and down-regulation of $m i R-221$ might be responsible for the anti-angiogenesis mechanisms of AS-IV and curcumin in HCC.

We previously demonstrated the inhibitory efficacy of Celastrus orbiculatus Thunb on the growth of HCC tumor through VEGF inhibition (10). The present report indicates that AS-IV and curcumin are active against HCC in orthotopic models, further indicating the potential of TCM for HCC. We also previously showed that AS-IV and curcumin were active in an orthotopic model of ovarian cancer (14).

In conclusion, this study shows that AS-IV and curcumin alone and in combination inhibit tumor growth and angiogenesis in an orthotopic nude-mouse model of human hepatocellular carcinoma. AS-IV and curcumin demonstrated synergistic suppressive efficacy against angiogenic factors VEGF, FGF-2, MMP-2 and HGF, as well as thrombosisrelated factors TF and FVII. Up-regulation of microRNAs $m i R-122$ and down-regulation of $m i R-221$ may mediate antiangiogenesis mechanisms of AS-IV and curcumin in HCC. This study indicates the potential for AS-IV and curcumin TCM for treatment of HCC.

\section{Conflicts of Interest}

The Authors declare no conflict of interest for this study.

\section{Acknowledgements}

This research was supported by the National Natural Science Foundation of China (no. 81373990). We also thank all our colleagues in our research group for their generous support. In addition, special thanks go to Prof. Jiangang Shen (School of Chinese Medicine, University of Hong Kong) for revision of this article.

\section{References}

1 Flores A and Marrero JA: Emerging trends in hepatocellular carcinoma: focus on diagnosis and therapeutics. Clin Med Insights Oncol 8: 71-76, 2014.

2 Wang WQ, Liu L, Xu HX, Luo GP, Chen T, Wu CT, Xu YF, Xu J, Liu C, Zhang B, Long J, Tang ZY and Yu XJ: Intratumoral $\alpha-$ SMA enhances the prognostic potency of CD34 associated with maintenance of microvessel integrity in hepatocellular carcinoma and pancreatic cancer. PLoS One 8: e71189, 2013.

3 Cheng X, Gu J, Zhang M, Yuan J, Zhao B, Jiang J and Jia X: Astragaloside IV inhibits migration and invasion in human lung cancer A549 cells via regulating PKC- $\alpha$-ERK1/2-NF- $k$ B pathway. Int Immunopharmacol 23: 304-313, 2014.
4 Wang ZS, Xiong F, Xie XH, Chen D, Pan JH and Cheng L: Astragaloside IV attenuates proteinuria in streptozotocin-induced diabetic nephropathy via the inhibition of endoplasmic reticulum stress. BMC Nephrol 16: 44, 2015.

5 Wilken R, Veena MS, Wang MB and Srivatsan ES: Curcumin: A review of anti-cancer properties and therapeutic activity in head and neck squamous cell carcinoma. Mol Cancer 10: 12, 2011.

6 Law PC, Auyeung KK, Chan LY and Ko JK: Astragalus saponins down-regulate vascular endothelial growth factor under cobalt chloride-stimulated hypoxia in colon cancer cells. BMC Complement Altern Med 12: 160, 2012.

7 Auyeung KK, Woo PK, Law PC and Ko JK: Astragalus saponins modulate cell invasiveness and angiogenesis in human gastric adenocarcinoma cells. J Ethnopharmacol 141: 635-641, 2012.

8 Lin SS, Lai KC, Hsu SC, Yang JS, Kuo CL, Lin JP, Ma YS, Wu $\mathrm{CC}$ and Chung JG: Curcumin inhibits the migration and invasion of human A549 lung cancer cells through the inhibition of matrix metalloproteinase-2 and -9 and vascular endothelial growth factor (VEGF). Cancer Lett 285: 127-133, 2009.

9 Yang CL, Liu YY, Ma YG, Xue YX, Liu DG, Ren Y, Liu XB, Li Y and Li Z: Curcumin blocks small cell lung cancer cells migration, invasion, angiogenesis, cell cycle and neoplasia through Janus kinase-STAT3 signalling pathway. PLoS One 7: e37960, 2012.

10 Wang M, Zhang X, Xiong X, Yang Z, Sun Y, Yang Z, Hoffman RM and Liu Y: Efficacy of the Chinese traditional medicinal herb Celastrus orbiculatus Thunb on human hepatocellular carcinoma in an orthotopic fluorescent nude mouse model. Anticancer Res 32: 1213-1220, 2012.

11 Zhang Y, Zhang N, Su S, Hoffman RM and Zhao M: Salmonella typhimurium A1-R tumor targeting in immunocompetent mice is enhanced by a Traditional Chinese Medicine herbal mixture. Anticancer Res 33: 1837-1843, 2013.

12 Zhang L, Wu C, Zhang Y, Liu F, Zhao M, Bouvet M and Hoffman RM: Efficacy comparison of traditional Chinese medicine LQ versus gemcitabine in a mouse model of pancreatic cancer. J Cell Biochem 114: 2131-2137, 2013.

13 Zhang L, Wu C, Zhang Y, Liu F, Wang X, Zhao M and Hoffman RM: Comparison of efficacy and toxicity of Traditional Chinese Medicine (TCM) herbal mixture LQ and conventional chemotherapy on lung cancer metastasis and survival in mouse models. PLoS One 9: e109814, 2014.

14 Yin G, Tang D, Dai J, Liu M, Wu M, Sun Y, Yang Z, Hoffman RM, Li L, Zhang S and Guo X: Combination efficacy of Astragalus membranaceus and Curcuma wenyujin at different stages of tumor progression in an orthotopic nude mouse model of metastatic human ovarian cancer. Anticancer Res 35: 31933208, 2015.

15 Scartozzi M, Faloppi L, Svegliati Baroni G, Loretelli C, Piscaglia F, Iavarone M, Toniutto P, Fava G, De Minicis S, Mandolesi A, Bianconi M, Giampieri R, Granito A, Facchetti F, Bitetto D, Marinelli S, Venerandi L, Vavassori S, Gemini S, D'Errico A, Colombo M, Bolondi L, Bearzi I, Benedetti A and Cascinu S: VEGF and VEGFR genotyping in the predict ion of clinical outcome for HCC patients receiving sorafenib: The ALICE-1 study. Int J Cancer 135: 1247-1256, 2014.

$16 \mathrm{Wu} \mathrm{Y,} \mathrm{Zheng} \mathrm{Y,} \mathrm{Shen} \mathrm{Z,} \mathrm{Ge} \mathrm{W,} \mathrm{Xie} \mathrm{Y} \mathrm{and} \mathrm{Li} \mathrm{C:} \mathrm{Endostar}$ combined with radiotherapy increases radiation sensitivity by decreasing the expression of TGF- $\beta 1$, HIF- $1 \alpha$ and bFGF. Exp Ther Med 7: 911-916, 2014. 
17 Haley EM and Kim Y: The role of basic fibroblast growth factor in glioblastoma multiforme and glioblastoma stem cells and in their in vitro culture. Cancer Lett 346: 1-5, 2013.

18 Stewart CJ and Crook ML: CD147 (EMMPRIN) and matrix metalloproteinase-2 expression in uterine endometrioid adenocarcinoma. Pathol Res Pract 207: 30-36, 2011.

19 Auyeung KK, Woo PK, Law PC and Ko JK: Astragalus saponins modulate cell invasiveness and angiogenesis in human gastric adenocarcinoma cells. J Ethnopharmacol 141: 635-641, 2012.

20 Di GH, Li HC, Shen ZZ and Shao ZM: Analysis of antiproliferation of curcumin on human breast cancer cells and its mechanism. Zhonghua Yi Xue Za Zhi 83: 1764-1768, 2003.

21 Rao B, Gao Y, Huang J, Gao X, Fu X, Huang M, Yao J, Wang $\mathrm{J}$, Li W, Zhang J, Liu H, Wang L and Wang J: Mutations of p53 and $K$-RAS correlate $T F$ expression in human colorectal carcinomas: TF down-regulation as a marker of poor prognosis. Int J Colorectal Dis 26: 593-601, 2011.

22 Chen L, Luo G, Tan Y, Wei J, Wu C, Zheng L, Zhang X and Xu $\mathrm{N}$ : Immunolocalisation of tissue factor in esophageal cancer is correlated with intratumoral angiogenesis and prognosis of the patient. Acta Histochem 112: 233-239, 2010.
23 Zerbib P, Grimonprez A, Corseaux D, Mouquet F, Nunes B, Petersen LC, Susen S, Ung A, Hebbar M, Pruvot FR, Chambon JP and Jude B: Inhibition of tissue factor-factor viia proteolytic activity blunts hepatic metastasis in colorectal cancer. J Surg Res 153: 239-245, 2009.

24 Coulouarn C, Factor VM, Andersen JB, Durkin ME and Thorgeirsson SS: Loss of miR-122 expression in liver cancer correlates with suppression of the hepatic phenotype and gain of metastatic properties. Oncogene 28: 3526-3536, 2009.

25 Tsai WC, Hsu PW, Lai TC, Chau GY, Lin CW, Chen CM, Lin CD, Liao YL, Wang JL, Chau YP, Hsu MT, Hsiao M, Huang HD and Tsou AP: MicroRNA-122, a tumor suppressor microRNA that regulates intrahepatic metastasis of hepatocellular carcinoma. Hepatology 49: 1571-1582, 2009.

26 Nicoli S, Knyphausen CP, Zhu LJ, Lakshmanan A and Lawson ND: miR-221 is required for endothelial tip cell behaviors during vascular development. Dev Cell 22: 418-429, 2012.

Received November 21, 2016

Revised December 16, 2016

Accepted December 20, 2016 